\title{
Treatment of Myoclonus
}

\author{
John N. Caviness
}

Published online: 14 September 2013

(C) The American Society for Experimental NeuroTherapeutics, Inc. 2013

\begin{abstract}
Myoclonus creates significant disability for patients. This symptom or sign can have many different etiologies, presentations, and pathophysiological mechanisms. A thorough evaluation for the myoclonus etiology is critical for developing a treatment strategy. The best etiological classification scheme is a modified version from that proposed by Marsden et al. in 1982. Clinical neurophysiology, as assessed by electromyography and electroencephalography, can be used to classify the pathophysiology of the myoclonus using a neurophysiology classification scheme. If the etiology of the myoclonus cannot be reversed or treated, then symptomatic treatment of the myoclonus itself may be warranted. Unfortunately, there are few controlled studies for myoclonus treatments. The treatment strategy for the myoclonus is best derived from the neurophysiology classification scheme categories: 1) cortical, 2) cortical-subcortical, 3) subcorticalnonsegmental, 4) segmental, and 5) peripheral. A cortical physiology classification is most common. Levetiracetam is suggested as first-line treatment for cortical myoclonus, but valproic acid and clonazepam are commonly used. Corticalsubcortical myoclonus is the physiology demonstrated by myoclonic seizures, such as in primary epileptic myoclonus (e.g., juvenile myoclonic epilepsy). Valproic acid has demonstrated efficacy in such epileptic syndromes with other medications providing an adjunctive role. Clonazepam is used for subcortical-nonsegmental myoclonus, but other treatments, depending on the syndrome, have been used for this physiological type of myoclonus. Segmental myoclonus is difficult
\end{abstract}

Electronic supplementary material The online version of this article (doi:10.1007/s13311-013-0216-3) contains supplementary material, which is available to authorized users.

J. N. Caviness $(\bowtie)$

Department of Neurology, Mayo Clinic Arizona, 13400 East Shea

Blvd., Scottsdale, AZ 85259, USA

e-mail: jcaviness@mayo.edu to treat, but clonazepam and botulinum toxin are used. Botulinum toxin is used for focal examples of peripheral myoclonus. Myoclonus treatment is commonly not effective and/or limited by side effects.

Keywords Myoclonus $\cdot$ Seizure $\cdot$ Epilepsy $\cdot$ Treatment . $\mathrm{EEG} \cdot \mathrm{EMG}$

\section{Introduction}

Myoclonus can be the cause of significant disability. Whether it is present at rest, with muscle activation, or from stimuli, myoclonic jerks interfere with performing or initiating the desired correct movement for a given task [1]. As a result, impairment in activities of daily living and intolerable frustration occur. Classically defined, myoclonus is a sudden, brief, lightening-like muscle jerk arising abnormally from the nervous system [2]. Any movement, of short or long-duration, that arises intrinsically from the muscle, such as fasciculation, spasm, or cramp, is not considered myoclonus. There is no strict rule for what duration constitutes the brevity threshold for myoclonus. However, the definition emphasizes how quickly the movement reaches its highest amplitude and then descends back to its original posture. Thus, myoclonic movements are the most sudden and brief muscle jerks that can be produced from involuntary nervous system stimulation. Nonmyoclonic jerks occur in dyskinesias such as chorea, ballismus, tardive dyskinesias, and dystonia.

Myoclonus occurs via many different etiologies and basic pathophysiological mechanisms [1]. Classification by both etiology and physiology is necessary to optimize the treatment strategy. Etiological classification is best performed by using a modified version of the scheme proposed by Marsden et al. [2]. A modified version is shown in Table 1. This classification system uses four main categories: physiologic, essential, 
Table 1 Etiology myoclonus classification scheme. Modified from Marsden et al. [2]

I. Physiologic myoclonus (healthy individuals)
A. Sleep jerks (e.g., hypnic jerks)
B. Anxiety-induced
C. Exercise-induced
D. Hiccough (singultus)
E. Benign infantile myoclonus with feeding

II. Essential myoclonus (primary symptom, non-progressive history)

A. Hereditary (autosomal dominant)

a. Myoclonus-dystonia syndrome (including $\varepsilon$-sarcoglycan mutations)

b. Other

B. Sporadic

III. Epileptic myoclonus (seizures dominate, part of chronic seizure disorder)

A. Fragments of epilepsy

a. Isolated epileptic myoclonic jerks

b. Focal motor seizures (includes epilepsia partialis continua)

c. Idiopathic stimulus-sensitive myoclonus

d. Photosensitive myoclonus

e. Myoclonic absences in petit mal epilepsy

B. Childhood myoclonic epilepsy

a. Infantile spasms

b. Myoclonic astatic epilepsy (Lennox-Gastaut)

c. Cryptogenic myoclonus epilepsy (Aicardi)

d. Awakening myoclonus epilepsy of Janz (juvenile myoclonic epilepsy)

C. Progressive myoclonus epilepsy: Baltic myoclonus

(Unverricht-Lundborg)

IV. Symptomatic myoclonus (secondary, progressive, or static encephalopathy dominates)

A. Storage disease
a. Lafora body disease
b. GM2 gangliosidosis (late infantile, juvenile)
c. Tay-Sachs disease
d. Gaucher disease (noninfantile neuronopathic form)
e. Krabbe leukodystrophy
f. Ceroid-lipofuscinosis (Batten)
g. Sialidosis (cherry-red spot) (types 1 and 2)

B. Spinocerebellar degenerations
a. Ramsay Hunt syndrome
b. Friedreich ataxia
c. Ataxia-telangiectasia
d. Other spinocerebellar degenerations

C. Basal ganglia degenerations
a. Wilson disease
b. Torsion dystonia
c. Hallervorden-Spatz disease
d. Progressive supranuclear palsy
e. Huntington disease
f. Parkinson disease

Table 1 (continued)
g. Multisystem atrophy
h. Corticobasal degeneration
i. Dentatorubropallidoluysian atrophy

A. Dementias

a. Creutzfeldt-Jakob disease

b. Alzheimer's disease

c. Dementia with Lewy bodies

d. Frontotemporal dementia (including mutations of Tau and others)

e. Rett syndrome

B. Infectious or postinfectious

a. Subacute sclerosing panencephalitis

b. Encephalitis lethargica

c. Arbovirus encephalitis

d. Herpes simplex encephalitis

e. Human T-lymphotropic virus I

f. HIV

g. Postinfectious encephalitis

h. Miscellaneous bacteria (Streptococcus, Clostridium, other)

i. Malaria

j. Syphilis

k. Cryptococcus

1. Lyme disease

m. Progressive multifocal leukoencephalopathy

A. Metabolic
a. Hyperthyroidism
b. Hepatic failure
c. Renal failure
d. Dialysis syndrome
e. Hyponatremia
f. Hypoglycemia
g. Nonketotic hyperglycemia
h. Multiple carboxylase deficiency
i. Biotin deficiency
j. Mitochondrial dysfunction
k. Hypoxia
1. Metabolic alkalosis
m. Vitamin E deficiency

B. Toxic and drug-induced syndromes (modified from [1])

a. Toxins

i. Bismuth

ii. Heavy metals

iii. Methyl bromide

iv. Dichlorodiphenyltrichloroethane

v. Organic compounds

b. Psychiatric medications [e.g., cyclic antidepressants, selective serotonin reuptake inhibitors, monoamine oxidase inhibitors, lithium, tardive syndrome (anti-psychotic use)]

c. Anti-infectious agents

d. Opiates 
Table 1 (continued)
e. Anti-seizure agents
f. Anesthetics
g. Contrast media
h. Cardiac medications (e.g., calcium channel blockers, antiarrhythmics)
i. Drug withdrawal
j. Other medications

B. Physical encephalopathies

a. Posthypoxia (Lance-Adams)

b. Post-traumatic

c. Heat stroke

d. Electric shock

e. Decompression injury

C. Focal nervous system damage

a. Vascular (ischemic, hematoma, etc.)

b. Post-thalamotomy

c. Tumor

d. Trauma

e. Inflammation (e.g., multiple sclerosis)

f. Developmental (e.g., "dysplasia")

g. Peripheral nervous system

D. Malabsorption

a. Celiac disease

b. Whipple disease

E. Eosinophilia-myalgia syndrome

F. Inflammatory and paraneoplastic encephalopathies

a. Opsoclonus-myoclonus syndrome

i. Idiopathic

ii. Paraneoplastic [ANNA-1 (anti-Hu), ANNA-2 (anti-Ri), absent antibodies]

iii. Infectious

iv. Other

b. Voltage-gated potassium channel antibody syndromes

c. N-methyl-D-aspartate receptor antibody encephalitis

d. Thyroperoxidase antibody syndromes

e. Ophelia syndrome

G. Exaggerated startle syndrome

a. Hereditary

b. Sporadic

H. Hashimoto encephalopathy

I. Multiple system degeneration

a. Allgrove syndrome

b. DiGeorge syndrome

c. Membranous lipodystrophy

J. Unknown or "idiopathic" (familial or sporadic)

epileptic, symptomatic. Each of these categories has certain characteristics and clinical presentations. Physiologic myoclonus occurs normally in people, but the degree to which it occurs varies between individuals. Both the clinical history and examination suggest normal circumstances. Myoclonus can occur as a normal phenomenon of sleep. "Hypnic jerks" occur as generalized jerks when falling asleep and represent one example of physiologic myoclonus. The normal startle jerk is another example. Essential myoclonus is mostly monosymptomatic, relatively non-progressive, and is usually associated with minor disability. A modern view of essential myoclonus acknowledges hereditary and sporadic forms, as well as other associated signs and symptoms [3]. Most notably, the hereditary myoclonus-dystonia syndrome is the best defined essential myoclonus entity. Epileptic myoclonus occurs in the setting of a chronic seizure disorder in which myoclonus is a major seizure phenotype. The myoclonic seizures in juvenile myoclonic epilepsy are a prime example of epileptic myoclonus. Symptomatic myoclonus, the largest category, consists of a variety of disparate etiologies [4]. All symptomatic myoclonus cases are secondary to a defined disorder, either neurologic or medical. Symptomatic examples of myoclonus are commonly associated with other neurological signs and symptoms, such as dementia, delirium, and other movement disorders. The placement of a specific myoclonus case into one of these four categories is the first recommended step towards diagnosis.

At this time, there is little evidence-based data available for myoclonus treatment. Two sources have discussed levels of evidence for myoclonus treatment in various instances $[5,6]$. Both of these sources designate the lower class or grade of evidence for most examples of treatment indication. For most myoclonus types, results of multiple placebo-controlled double-blind studies are not available. Many articles on the treatment of myoclonus are case reports or small series. This article will focus on current practical treatment strategies. It has been established that delineation of myoclonus physiology is critical for the formulation of specific and successful myoclonus treatment strategy $[1,6]$. From the standpoint of the individual myoclonus patient, a treatment strategy is synthesized from etiology classification, physiology classification, best available evidence, and the patient-specific clinical characteristics of the myoclonus that create disability.

\section{Pathophysiology and Neurophysiological Classification of Myoclonus}

The pathophysiology of myoclonus, as well as its modern physiologic classification, refers to not only the site(s) of the abnormal neuronal circuits believed to create the myoclonus, but is also combined with the physiologic mechanism that generates and/or propagates the excitation. Currently, we do not know the exact neuronal circuits that are abnormal in any example of myoclonus. However, clinical 
neurophysiology testing is the best tool for gathering evidence to formulate physiological classification of myoclonus $[7,8]$. This testing commonly uses electroencephalography (EEG), surface electromyography (EMG), EEGEMG polygraphy with back-averaging, evoked potentials (most commonly somatosensory evoked potentials), and EMG responses to stimulation. Other techniques include coherence analysis and transcranial magnetic stimulation. Results from the test battery are used to classify the myoclonus according to a physiological classification for myoclonus. Here, the defining characteristics for each physiological classification are discussed. In the section on treatment, other physiological aspects will be given as they directly relate to treatment mechanism (s) under the categories. Categories of this classification scheme are:

- cortical;

- cortical-subcortical;

- subcortical-nonsegmental;

- segmental;

- peripheral.

\section{Cortical Myoclonus}

The cerebral cortex is the most common origin for myoclonus. The jerks are most often multifocal, but focal, segmental, and generalized myoclonus may also occur. Action myoclonus, i.e., myoclonus exacerbated/triggered by muscle activation, is common. As functional ability depends on precise muscle activation, overall treatment benefit often depends on the reduction of action myoclonus. An example of cortical myoclonus surface EMG polygraphy is given in Fig. 1. In Fig. 1, multiple multifocal short-duration hypersynchronous myoclonus EMG discharges may be appreciated with co-contraction of agonists and antagonists, and across muscle segments. To satisfy criteria for cortical myoclonus, there must be a focal time-locked cortical transient demonstrated that precedes the myoclonus by a short latency ( $<40 \mathrm{~ms}$ for arm). Although this may be observed on gross EEG-EMG polygraphy, backaveraging is a more sensitive and reliable method to derive EEG transients time-locked to myoclonus from ongoing EEG activity. EEG back-averaging of the myoclonus EMG discharges from Fig. 1 is shown in Fig. 2. Such EEG transients

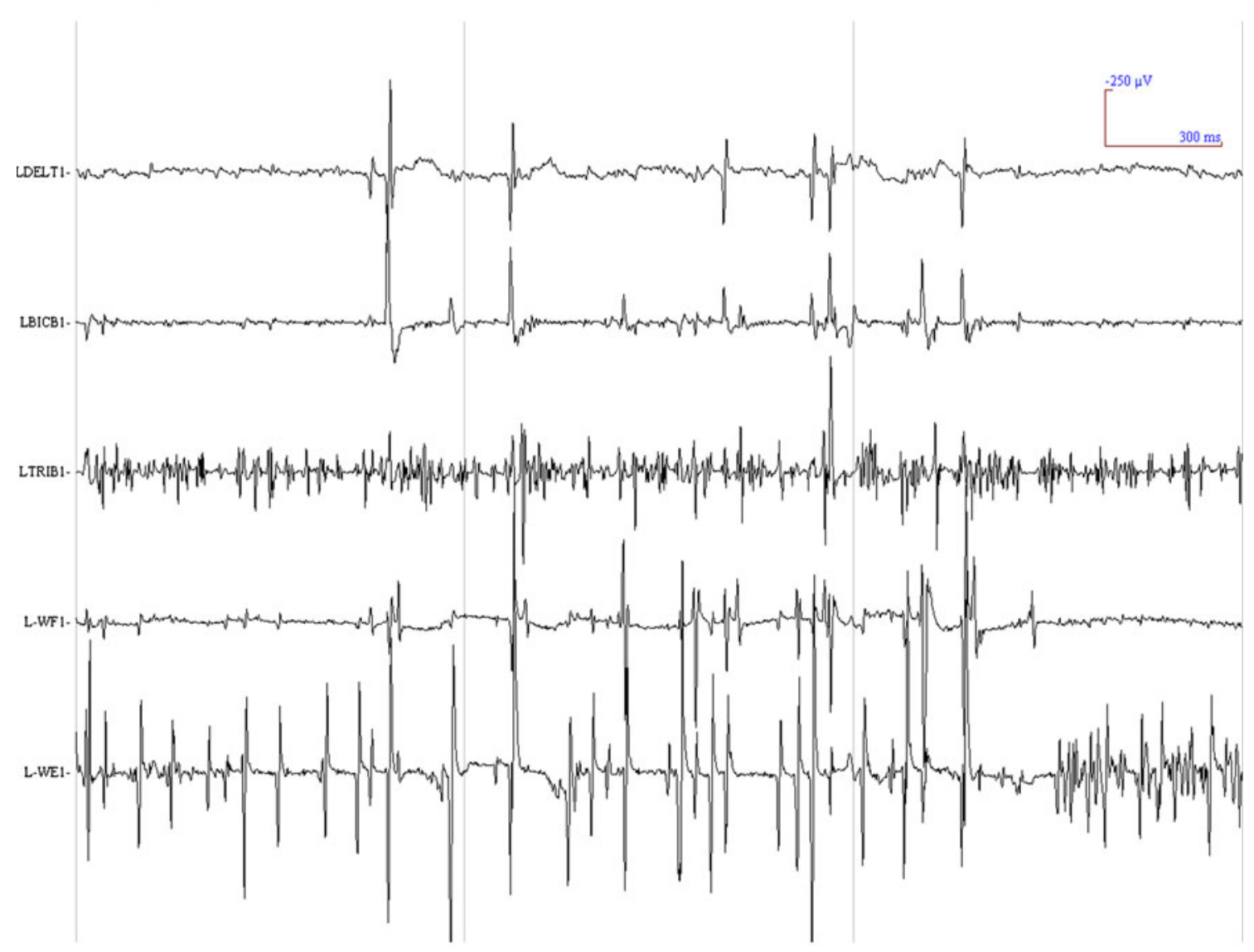

Fig. 1 Surface electromyography polygraphy recording from multiple left arm muscles in a patient with cortical myoclonus during muscle activation. Note the hypersynchronous and multifocal nature of the discharges that commonly occur with co-contraction of agonists and antagonists, sometimes across muscle segments. The electroencephalography back-averaging of these discharges is shown in Fig. 2 
typically have a focal distribution with a biphasic or triphasic spike waveform beginning with a positive deflection that precedes the onset of the myoclonic discharge by $6-22 \mathrm{~ms}$ in the upper extremity: the more distal muscle the myoclonus is recorded from, the longer the time interval [7]. The duration of the transient is $15-40 \mathrm{~ms}$. Compared with the spike amplitude seen on the EEG in partial epilepsy, amplitude of the spike that is time-locked to the myoclonus is small, often 5-20 $\mu \mathrm{V}$. The conduction of the spike to motor neuron pools is presumed to occur by fast-conducting corticospinal (pyramidal) pathways. The maximum of the transient is usually located over the sensorimotor cortex at the central or centro-parietal electrode according to anatomical somatotopic mapping, contralateral to the myoclonus. Enlarged cortical SEP waves and enhanced long latency EMG responses to electrical nerve stimulation are not uniformly present, but support a cortical origin for myoclonus. Elevated cortico-muscular coherence of the myoclonus EMG discharge that localizes to the contralateral sensorimotor cortex supports a cortical origin for myoclonus. Examples of cortical myoclonus occur post-hypoxia ("Lance-Adams syndrome"), lipid storage disorders, dementia syndromes, Parkinson's disease, and certain drug-induced etiologies such as lithium treatment.

\section{Cortical-Subcortical Myoclonus}

There is strong evidence that some generalized seizure phenomena arise from paroxysmal abnormal excessive oscillation in bidirectional connections between cortical and subcortical structures. This concept is supported by studies on animal models, neurotransmitter biochemistry, and imaging studies
[9-12]. The term "cortical-subcortical" myoclonus refers to myoclonus arising from this type of physiology [13]. Compared with a single discharge of cortical myoclonus, the abnormal excessive reciprocal excitation of cortical and subcortical sites is much more diffuse and bilateral at the instant of myoclonus generation. Although the subcortical involvement increases the excessive excitation, it is the spreading of excitation to the cortical motor centers that drives the myoclonus event. It is not exactly known what pathways, or possibly other subcortical sites, relay/produce this motor excitation. This myoclonus usually occurs in paroxysms from rest and clinically manifest as generalized seizures with a myoclonic component. As the cortical area excitation is diffuse and beyond motor areas, other clinical seizure phenomena, such as alteration of consciousness, may occur. The myoclonus is often generalized or bilaterally synchronous, but focal or multifocal distributions also occur. The cortical correlate is that of fast or slow spike and wave EEG discharges. Commonly, spike or polyspike discharges are seen. Surface EMG myoclonus discharges may be just as brief as in cortical myoclonus or a little longer (up to $100 \mathrm{~ms}$, approximately). Cortical SEP waves have been found to be enlarged by some investigators, but this is not a consistent finding. Examples of cortical-subcortical myoclonus occur in juvenile myoclonic epilepsy and absence seizure disorders. In the example of juvenile myoclonic epilepsy, the cortical-subcortical physiology is the myoclonic seizure accompanied by generalized EEG spike and wave discharges with upper body generalized jerks \pm head/limb involvement. For absence seizure disorders, the myoclonic jerk is sometimes an accompaniment to the alteration in consciousness, but the

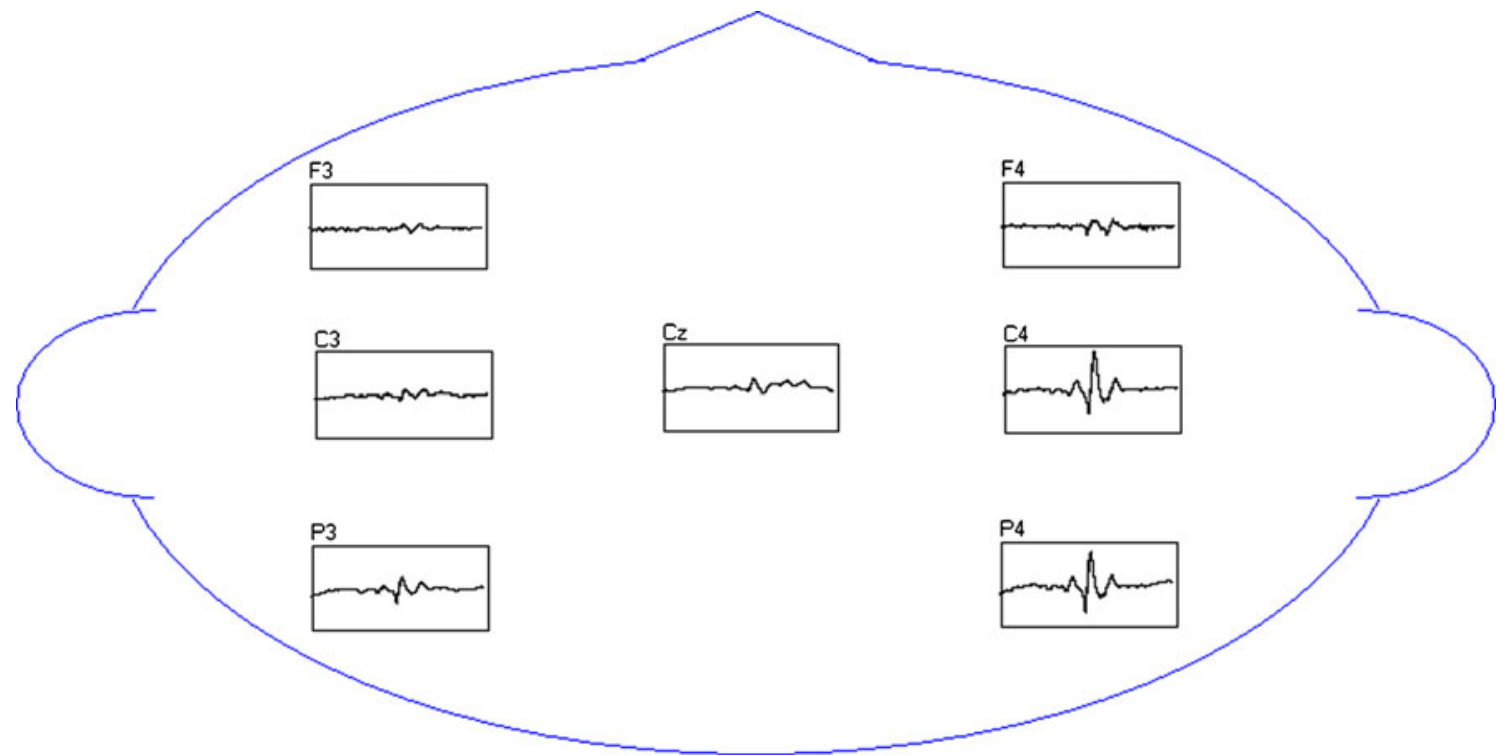

Fig. 2 Electroencephalography (EEG) back-averaging of the surface electromyography discharges that are shown in Fig. 1. The EEG transient has a right centro-parietal maximum, consistent with left arm cortical myoclonus. The EEG transient begins $24 \mathrm{~ms}$ before the $0 \mathrm{~ms}$ trigger point, consistent with corticospinal transmission of the myoclonus generation. The $x$-axis is $200 \mathrm{~ms}$ across left to right full extent. The $y$-axis is $100 \mu \mathrm{V}$ bottom to top full extent 
generalized EEG spike and wave discharges accompany the upper body myoclonic movements.

\section{Subcortical-Nonsegmental Myoclonus}

The clinical and neurophysiological characteristics of subcortical myoclonus are more variable than for those in cortical or cortical-subcortical myoclonus. This variability is in no doubt owing to the multiple possible subcortical sites, nuclei, and neuronal circuits from which a burst of excessive activity may arise and be transmitted to descending motor pathways. The myoclonus EMG duration may be longer (up to $200 \mathrm{~ms}$ ) than in cortical or cortical-subcortical myoclonus, but there are exceptions. Simultaneous rostral and caudal recruitment of the myoclonus from a subcortical site, which is reflected in the EMG channels, supports a subcortical generator. In other instances, the EMG recruitment order is strictly descending or coincides with a multifocal distribution. Abnormal cortical excitability does not drive or correlate with this myoclonus, so clinical neurophysiology methods do not show such abnormalities (e.g., EEG spikes, enlarged cortical SEP waves). Assignment of a case to the subcortical category can be problematic if it is based largely on absence of evidence for abnormal cortical excitability. This designation does not include segmental myoclonus generation as the pathophysiology and characteristics of segmental myoclonus are distinct. Some examples of subcortical-nonsegmental myoclonus with their putative source locations in parentheses are: myoclonusdystonia syndrome (basal ganglia), opsoclonus-myoclonus syndrome (brainstem-cerebellar circuits), reticular reflex myoclonus (brainstem reticular formation), and propriospinal myoclonus (spinal cord) [14-16].

\section{Segmental Myoclonus}

Segmental myoclonus has its generator at a particular segment or contiguous segments of the brainstem and/or spinal cord. This segmental generator produces movements at a particular segment or few contiguous segments, giving a focal or circumscribed distribution. There is usually persistent, rhythmical activation of muscles corresponding to the brainstem/spinal segment(s) involved. This myoclonus is known to be relatively unaffected by state of consciousness, motor activity, or stimulus. However, a more irregular timing with stimulus sensitivity sometimes occurs. The most common type of segmental myoclonus is palatal myoclonus followed by spinal segmental myoclonus. When the palatal movements are very regular, some authors use the less common term "palatal tremor". In spinal segmental myoclonus, the surface EMG shows synchronous rhythmic or semi-rhythmic discharges in muscles supplied by the corresponding spinal segmental generator [17]. The polygraphic surface EMG study usually shows synchronous activation of the affected muscles. The typical frequency is in the range of 1-3 Hz with a broad reported range of 0.2$8 \mathrm{~Hz}$, and the typical surface EMG discharge duration varies widely between 50 and $500 \mathrm{~ms}[18,19]$.

\section{Peripheral Myoclonus}

Peripheral myoclonus refers to myoclonic jerks that are driven from a peripheral site. Such EMG discharges are characterized by marked duration variability from discharge to discharge. The appearance and timing of EMG discharges, which are supplied by the same nerve, usually resemble each other. It is often difficult to separate motor excitation that arises from the peripheral nervous system versus a peripheral lesion that alters central nervous system motor centers and pathways. The bestdocumented example of peripheral myoclonus is hemifacial spasm.

\section{Basic Treatment Strategy for Myoclonus}

The treatment of myoclonus is often challenging. Myoclonus occurs in so many different forms and disorders such that a simple and straightforward recipe for treatment does not exist. Multiple treatments have been tried for myoclonus, but results are often inconsistent. Disabling side effects are common. Polypharmacy is common in myoclonus treatment, but it has many pitfalls. Because of these many challenges, it is necessary to have a treatment strategy that derives from the best diagnosis and physiological information that is available. Figure 3 shows a treatment algorithm for myoclonus. It begins with clinical history and examination, and appropriate testing. Depending on the evaluation results, treatment is tailored to the circumstance and physiological classification. All treatments outlined in Fig. 3 should be considered "off-label" and based on few, if any, controlled treatment trials. As such, clinical judgment must be exercised. Details and explanation are given below.

The diagnosis of myoclonus may be fairly certain because of the medical circumstances, genetic test results, etc. However, the determination of the diagnosis responsible for the myoclonus is not always clear and may require extensive investigation. If the etiology is unclear, then a rigorous diagnostic evaluation should be planned [1]. First, the clinical presentation category of the etiology classification scheme should be determined to be either physiologic (normal), essential (sporadic or hereditary), epileptic, or symptomatic. Second, basic testing is undertaken with easily available methods. These include central nervous systemic imaging, blood and urine testing for toxic metabolic causes, and antibody testing for inflammatory and para-neoplastic disorders. However, the absence of para-neoplastic antibodies should not be assumed to rule out neoplastic presence. Third, clinical 


\section{Myoclonus}

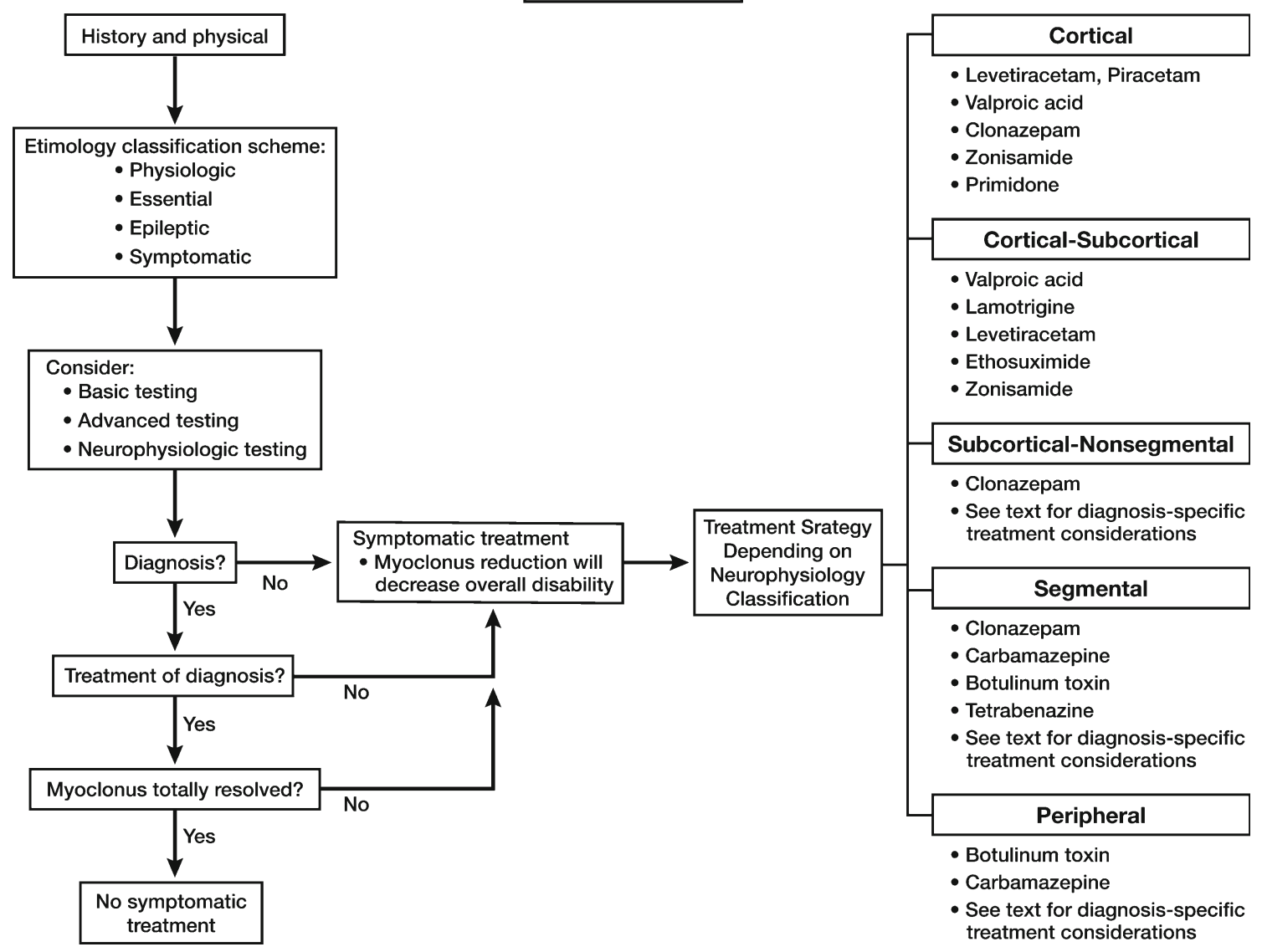

Fig. 3 Myoclonus treatment algorithm. See text for approach and details

neurophysiology testing with EEG and surface EMG should be performed. If a cortical source is possible, SEPs, EMG responses to stimulation, and referral to a laboratory that performs EEG-EMG polygraphy with back-averaging is recommended. Finally, depending on the clinical scenario and results so far available, advanced testing with a variety of established and emerging methods may be warranted. These may include, but are not limited to, tissue diagnosis, genetic testing, cerebrospinal fluid examination, enzyme and other biochemical testing, other imaging, and malabsorption testing.

The first consideration should be given to reversing the underlying etiology of the myoclonus. The most straightforward example is that of drug-induced myoclonus, and discontinuation of the drug usually eliminates the myoclonus. Lithium and antidepressant medication are two examples [20]. Other potentially reversible causes of myoclonus are an acquired abnormal metabolic state, removable toxin, or an excisable lesion. Some cases of propriospinal myoclonus have a lesion affecting the spinal cord that correlates with the origin from which the myoclonus EMG discharges spread. In such instances, it is possible for the propriospinal myoclonus to be partially or totally relieved by lesion removal [21]. Paraneoplastic myoclonus has also been known to improve, on occasion, if the cancer is treated effectively. Before treating any etiology, the risks need to be considered for the patient. Nevertheless, owing to the limitations of symptomatic therapy, the treatment of the underlying etiology may be the best option if the clinical circumstances are appropriate.

For the majority of myoclonus cases, treatment of the underlying disorder is usually not possible or effective, and symptomatic treatment is justified if the myoclonus is disabling enough. Before making the decision to treat on a symptomatic basis, the clinician needs to consider whether potential side effects outweigh potential benefit. One example is a patient with significant dementia and myoclonus. Although the myoclonus may be suppressed with therapy, sedation and increased cognitive difficulty may well not be worth the decreased myoclonus. Another example is the generalized myoclonus that can occur immediately after prolonged hypotension due to cardiac arrest. This myoclonus is difficult to treat in a nonaggressive manner. It may be suppressed by anesthesia or paralytic agents temporarily, but there is risk in doing this. In some circumstances of myoclonus treatment, multiple drug combinations may be necessary for symptomatic treatment but also may increase side effects. Amelioration of the myoclonus is rare for 
symptomatic treatment. However, important improvement may be attained with decreased disability.

If the risks are judged to be warranted clinically, the best strategy for symptomatic treatment is derived from using physiological classification. A drug treatment used for one physiological classification may not work well in another or may even induce worsening. For example, a medication that can work in some instances of segmental myoclonus may not work in cortical myoclonus. If the myoclonus physiology classification cannot be determined, then presuming the myoclonus physiology that usually occurs in that particular diagnosis is a reasonable way to cautiously proceed. If both the diagnosis and myoclonus physiology are unknown, there is little to guide an approach to treatment. In this instance, the clinician should be prepared for possible poor results until the reason for the myoclonus is defined. The myoclonus treatment strategy given below is organized by the physiological classification of the myoclonus.

\section{Cortical Myoclonus Treatment}

Suppression of myoclonus may come from lowering the excitatory drive and/or increasing inhibition of the responsible neurons that drive the excitatory output. Two observations suggest cortical myoclonus arises from abnormal excitation of corticospinal output: 1) cortical myoclonus shows a timelocked pre-myoclonus EEG discharge reflecting apical dendrite excitation of pyramidal neurons; 2) myoclonus event latency is consistent with pyramidal tract conduction. Common occurrence of multifocal activation is allowed by hyperexcitability across the relatively large sensorimotor cortex homunculi. Such hyperexcitability may be exacerbated by voluntary or reflex activation. Within this region, large pyramidal neurons of layers III and V are presumed to be subject to the cortical hyperexcitability that causes excessive and brief myoclonus discharges down their axons [22]. Decreasing the excitatory drive of these neurons and their circuits may come from altering ionic conductance or decreasing excitatory neurotransmission, such as via the neurotransmitter glutamate system. Chandelier, basket, and other gamma-aminobutyric acid (GABA)-ergic neurons are powerful sources of inhibition for pyramidal cortical neurons and associated circuits. Enhanced inhibitory activity through these neurons and/or their GABA-ergic receptors has a potential therapeutic effect for cortical myoclonus. For treatment of cortical myoclonus to be effective, it must affect these intrinsic circuits within the sensorimotor cortex.

Levetiracetam, Piracetam, and Related Compounds

Levetiracetam and piracetam are related drugs and have had limited controlled study for myoclonus treatment [23-28].
Their mechanism of action remains unknown, but their binding to the synaptic vesicle protein $2 \mathrm{~A}$ (SV2A) may moderate neurotransmitter release. Interaction does not occur with GABA or glutamate receptors. Among this class of compounds, the degree of SV2A binding correlates with an anti-seizure effect. In addition, levetiracetam modulates $\mathrm{Ca}^{2+}$ and $\mathrm{K}^{+}$currents. Therefore, these compounds may reduce hyperexcitability without altering normal activity by preventing abnormally increased neurotransmitter exocytosis.

Both levetiracetam and piracetam are relatively welltolerated and non-sedating, but psychiatric, gastrointestinal, and other side effects can occur. Because of their demonstrated effectiveness and relatively favorable side effect profile, these drugs are recommended as initial therapy. There are anecdotal and small series reports for levetiracetam responsiveness in many of the specific etiologies of cortical myoclonus. Daily dosages of levetiracetam range between $1000 \mathrm{mg}$ and $3000 \mathrm{mg}$, and for piracetam range between $2.4 \mathrm{~g}$ and $21.6 \mathrm{~g}$. Abrupt withdrawal may precipitate a severe worsening of myoclonus and, in the case of piracetam, seizures may occur.

Brivaracetam is one of the new compounds that are derivatives of levetiracetam. It has a higher affinity for the SV2A protein than levetiracetam, and inhibits $\mathrm{Na}^{+}$currents and $\mathrm{N}$ methyl-D-aspartate glutamate receptors. This agent is still considered experimental, but evidence of its effectiveness in partial epilepsy is increasing. In an animal model of posthypoxic myoclonus, brivaracetam was more potent than levetiracetam [29]. Results in Unverricht-Lundborg disease showed an anti-myoclonus effect [30]. Much more evidence is needed, but brivaracetam has the potential to add to myoclonus therapeutics.

\section{Sodium Valproate}

The therapeutic action of valproic acid in cortical myoclonus is believed to occur through augmentation of the inhibitory influence of GABA. This occurs through multiple mechanisms, including increased synthesis and decreased degradation of GABA. Valproate acid also has some ability to decrease excitation by glutamate and alter ionic conductance [31]. Many patients need doses of 1200-2000 mg/day for myoclonus treatment [32]. Transient gastrointestinal upset may occur during initial treatment, usually with nausea and vomiting, but sometimes with abdominal pain and diarrhea. Hair loss, tremor, hepatotoxicity, and drowsiness may also occur. A recent report has provided insight about the nature of occasionally induced parkinsonism by valproic acid [33]. Such instances may be insidious but still reversible by drug discontinuation, so continual monitoring for side effects is important. 


\section{Clonazepam}

For cortical myoclonus, clonazepam is a useful adjunct to treatment by levetiracetam or valproic acid. However, clonazepam occasionally works as a monotherapy. The predominant mechanism for the anti-myoclonic action of clonazepam is thought to be the enhancement of GABAergic neurotransmission via benzodiazepine receptors [34]. Benzodiazepine receptors are diffusely distributed throughout the brain which may explain this drug's ability to treat myoclonus in noncortical locations as well. Clonazepam has been found to increase serotoninergic neurotransmission, but the mechanism is far from clear $[35,36]$. Clonazepam has an advantage in that it has a longer duration of action than other benzodiazepines, such as diazepam or alprazolam. Clonazepam doses may need to be high to achieve treatment effect (as much as $6 \mathrm{mg} /$ day or more) but should be introduced slowly [32]. Drowsiness and ataxia are major side effects and can be sometimes overcome by gradually increasing the dosage. Abrupt reductions and withdrawals can result in a marked deterioration in myoclonus and withdrawal seizures. Tolerance may develop over a period of several months and is, unfortunately, common.

\section{Zonisamide, Primidone, and Miscellaneous Agents}

Most of the other numerous agents that have been used to treat cortical myoclonus are anti-seizure medications. Although the exact mechanisms of these other agents are poorly understood, presumably it is their ability to suppress excessive neuronal firing within myoclonus-generating circuits that suppresses the myoclonus when they are effective. Zonisamide has been reported to help when other add-on agents have failed [37]. Primidone and phenobarbital can be useful as add-on therapy [32]. Phenytoin and carbamazepine are helpful in only a minority of patients and, in fact, may actually exacerbate myoclonus, so they have little, if any, value in the present-day treatment of cortical myoclonus except in the case of partial motor seizures. These examples serve as a useful reminder that anti-seizure medications, both old and new, have the potential to worsen myoclonus in certain patients. Sodium oxybate, the sodium salt form of gamma-hydroxybutyric acid, has been reported to decrease cortical myoclonus in a number of patients [38]. Sodium oxybate has serious side effects, including respiratory suppression, so caution is warranted [38].

\section{Cortical-Subcortical Myoclonus Treatment}

The myoclonus in primary generalized epilepsies falls under this physiological classification, including myoclonic seizures. In primary generalized epilepsies, the abnormally activated circuit is comprised of neocortical pyramidal neurons, thalamic relay neurons, and the GABAergic neurons of the nucleus reticularis thalami [39]. This network is widely distributed; when synchronized burst-firing occurs within it, generalized epileptic seizures can manifest. Myoclonus may occur if such burst-firing affects motor cortex. Treatment of cortical-subcortical myoclonus must decrease the reverberating excitatory activity of these cortical-subcortical circuits. $\mathrm{GABA}_{\mathrm{A}}$ and $\mathrm{GABA}_{\mathrm{B}}$ activation, as well as $\mathrm{Na}^{+}$and $\mathrm{Ca}^{2+}$ conductance, are mechanisms that are believed to play a crucial role in burst-firing [40]. Mutations of proteins associated with GABA receptors and ionic conductance are known to produce myoclonic seizures and other generalized epilepsies [41]. Treatments that intercede in these mechanisms have potential for a therapeutic action for cortical-subcortical myoclonus.

Valproic acid is the major drug of choice for corticalsubcortical myoclonus overall [42]. However, the controlled evidence for efficacy is mostly for juvenile myoclonic epilepsy $[43,44]$. Less impressive results are seen in other childhood myoclonic epilepsy syndromes [45]. Why is valproic acid seemingly more effective for cortical-subcortical myoclonus than levetiracetam, while the relative efficacy is reversed for cortical myoclonus? Both agents do have treatment efficacy for both physiological types of myoclonus. However, as aberrant ionic conductance (e.g., $\mathrm{Na}^{+}$and $\mathrm{Ca}^{2+}$ ) plays a substantial role in cortical-subcortical myoclonus pathophysiology, the ability of valproic acid to affect ionic conductance may be more significant than for levetiracetam [46]. The precise reason is not known. Lamotrigine may be used alone or as an adjunct to valproic acid [46]. The role for ethosuximide, zonisamide, and clonazepam are mainly as adjuncts [42]. Polypharmacy may be useful, but is limited by side effects. Paradoxically, anti-seizure medications sometimes increase seizures or myoclonus in these syndromes, including phenytoin, carbamazepine, and lamotrigine [47-49]. Intravenous valproic acid or levetiracetam can be useful in myoclonic seizure status [50]. Apart from the myoclonus per se, the treatment of both primary and secondary generalized myoclonic epilepsy syndromes has extensive algorithms organized around age of onset and subtypes [51-53].

\section{Subcortical-Nonsegmental Myoclonus Treatment}

The mechanisms of subcortical-nonsegmental myoclonus are heterogeneous. Moreover, the aberrant circuitry for these myoclonus examples is even less understood than it is for cortical myoclonus. The therapeutics of this myoclonus physiology overlaps that of cortical myoclonus, but also has important differences. Although GABAergic mechanisms can be leveraged in these myoclonus disorders, cholinergic and monoamine systems also seem to offer an opportunity for therapeutics. Standard anti-seizure treatments, such as valproic acid and 
levetiracetam, are not as consistently useful in subcorticalnonsegmental myoclonus when compared with their use in cortical or cortical-subcortical myoclonus.

\section{Myoclonus-Dystonia Syndrome}

The myoclonus-dystonia syndrome, also referred to as essential myoclonus, is thought to have its circuit abnormality in the basal ganglia. The basal ganglia possess GABAergic pathways, and, presumably, clonazepam performs its therapeutic action on myoclonus-dystonia through those pathways $[34,54]$. The nuclei of the basal ganglia are believed to be the brain origin where the circuit abnormality may exist. It is perhaps the cholinergic pathways within the basal ganglia that are responsible for the reported therapeutic action of anti-cholinergic medication in myoclonus-dystonia [55]. Sodium oxybate, the sodium salt form of gamma-hydroxybutyric acid, has been reported to decrease myoclonus in a few cases of myoclonus-dystonia [38]. Sodium oxybate has serious side effects, including respiratory suppression, so caution is warranted [38]. Deep brain stimulation of the thalamus or globus pallidus has had success in case reports and awaits confirmation in a larger number of patients [56].

\section{Opsoclonus-Myoclonus Syndrome}

The opsoclonus-myoclonus syndrome has a dramatic presentation and has been ascribed to brainstem and cerebellar pathways [54]. Inflammatory, infectious, and antibodyassociated disorders are prominent causes of the opsoclonus-myoclonus syndrome. Owing to the nature of these etiologies, it is understandable that some patients are responsive to intravenous immunoglobulin therapy, plasmaphereis, and steroid-based strategies [57]. In these cases, it would appear that these treatments may be affecting the underlying autoimmune disorder rather than the myoclonus per se. Paraneoplastic cases are important to recognize as the movement disorder may be the major initial clinical presentation of the cancer [54, 58]. Sometimes, treatment of the cancer can be associated with secondary improvement of the myoclonus. In childhood, the opsoclonus-myoclonus syndrome may be paraneoplastic and associated with a neuroblastoma. Whether associated with cancer or not, treatment considerations in children differ from the adult form. Clonazepam has been reported to be useful in some cases [54]. Other treatments reported to be useful in certain cases include valproic acid, piracetam, and reserpine [58-60].

Reticular Reflex Myoclonus

Reticular reflex myoclonus is rare. Most often, it is seen in the setting of post-hypoxia and uremia. Uncommonly, it occurs chronically after recovery from post-hypoxia. The generalized myoclonus that may occur almost immediately post-hypoxia resembles reticular reflex myoclonus. Generalized EEG discharges may accompany the myoclonus, but it is often unclear whether this represents seizures or "status myoclonus". If the patient recovers from the hypoxia, or in the case of uremia be reversed by dialysis, then this myoclonus improves. If symptomatic treatment is warranted, clonazepam is reported to be effective. If a dramatic response is needed, clonazepam may be given intravenously with appropriate precautions taken to monitor and support vital signs if needed [16]. 5-Hydroxytryptophan was initially reported as helpful, but is now used rarely, if at all [61].

\section{Propriospinal Myoclonus}

Propriospinal myoclonus has been associated with focal lesions that when treated with surgical removal may resolve the myoclonus [21]. For this reason, complete spinal cord imaging, such as with magnetic resonance imaging, is critically important. The neurophysiological finding of surface EMG discharges arising first at a specific spinal cord level can provide corroborative evidence for the myoclonus origin and, possibly, the lesion causing it. Clinical judgment is needed to determine whether removal could reverse a lesion effect, but spinal cord damage may remain, even after structural lesion removal. Clonazepam is the first line of symptomatic therapy for propriospinal myoclonus. Zonisamide has been reported to be effective in some cases [62]. Other therapies that have been reported in the literature include lioresal, valproic acid, and carbamazepine [63]. It should be realized that psychogenic jerks (discussed below) can be commonly misdiagnosed as propriospinal myoclonus [64]. If the clinician believes this to be a possibility, then it is wise to consider changing the diagnosis and treating accordingly.

\section{Segmental Myoclonus Treatment}

Segmental myoclonus is generated at a circumscribed level along the brainstem and/or spinal neuraxis. Often, it is produced by a local lesion causes a focal neuronal circuit disruption with motor neurons having an abnormal bursting discharge pattern [17]. This lesion may occur in the gray matter of the spinal cord or in the central region of the brainstem reticular formation while preserving motorneuron output. A disconnection of afferent white matter tracts that project to nuclei or gray matter that, in turn, project to motor neurons can also generate the bursting discharge pattern of segmental myoclonus. At a segmental level, important inhibitory circuits are GABAergic and/or glycinergic, and these are potential mechanisms on which to base symptomatic treatment. 
Descending monoaminergic pathways also seem to play a role in modulating motor activity. These neurotransmitter systems provide a possible basis to suppress the segmental myoclonus bursting pattern. A variety of lesions may cause segmental myoclonus but vascular, tumor, trauma, infectious, and idiopathic and/or "essential" diagnoses account for most cases.

\section{Palatal Segmental Myoclonus}

An amazing variety of medications has been used for treating palatal myoclonus and all are "off-label". This is, in part, owing to the difficultly in achieving a satisfactory treatment effect. The drugs with anecdotal success in palatal myoclonus include clonazepam, carbamazepine, lioresal, anticholinergics, tetrabenazine, valproic acid, phenytoin, lamotrigine, sumatriptan, 5-hydroxytryptophan, and piracetam [65-69]. Both drug-specific side effects and those common among several medications are often dose-limiting. Therefore, caution is advised during titration. The medications on this list have the capability to augment GABAergic or glycinergic inhibition and/or favorably modulate monoaminergic neurotransmission. When ear-clicking is disabling to the patient, surgical treatments such as tensor veli palatini tenotomy and occlusion of the Eustachian tube may be performed. However, success has been variable [70]. Botulinum toxin injections have been reported to be useful, and the literature on this treatment is increasing [17, 71-73]. However, important principles should be realized: 1) botulinum toxin is not yet proven safe or effective in palatal myoclonus; 2) injections should be performed by a qualified ear, nose, and throat surgeons; 3 ) electromyography guidance is considered useful; and 4) patients should be informed about the uncertainty/side effects of this therapy [17].

\section{Spinal Segmental Myoclonus}

Treatment of spinal segmental myoclonus is no less challenging than that for palatal myoclonus and also "off-label". Clonazepam, in dosages up to $6 \mathrm{mg}$ daily, is commonly tried first in spinal segmental myoclonus, but complete suppression of the myoclonus is uncommon [1]. Levetiracetam is reported to decrease the movements $[74,75]$. Lioresal, carbamazepine, and topiramate have worked in some instances [55, 76]. Tetrabenazine can be useful in cases refractory to other treatments [65]. Botulinum toxin injections may treat the pain or movements associated with spinal segmental myoclonus [77]. AS the disease process causing the myoclonus sometimes evolves and has a natural history to remit, one must be careful in determining that a presumably symptomatic treatment cures the underlying pathophysiological process.

\section{Peripheral Myoclonus Treatment}

The myoclonic movements of hemifacial spasm will often respond to botulinum toxin therapy along with the more tonic or sustained contractions [77]. Surgery to remove pressure on the seventh cranial nerve may be warranted in certain situations. Other causes of peripheral myoclonus may also respond to botulinum toxin injections, and this may also improve any associated discomfort with the movements [78]. Carbamazepine may be tried as drug therapy for peripheral myoclonus, but a satisfactory response is uncommon.

\section{Treatment of Psychogenic Jerks}

Psychogenic or "functional" jerks are diagnosed when the movements are thought to be a symptom of psychiatric disease [79]. The term "psychogenic jerk" is preferred to "psychogenic myoclonus" because the movements are usually not typical for myoclonus and often are longer or more complex. Psychogenic jerks offer diagnostic and treatment challenges. For example, it has been pointed out that psychogenic jerks are commonly misdiagnosed as propriospinal myoclonus [64]. The diagnostic approach to psychogenic jerks may include neurophysiologic studies, but these are not always possible or definitive, so diagnosis strongly rests on the psychiatric aspects of the patient and the atypical nature of the movements. Treatment should begin with explaining to the patient the nature of the disorder. When in the context of nonmalingering, the patient should be told that the jerks are not their fault and that successful treatment is possible. A psychiatrist should consider treatment of any psychiatric disease that is present, such as anxiety and/or depression. Both physical therapy-based and cognitive behavioral therapies have been reported to be useful [80]. The clinician should keep in mind that each of these cases tends to be unique, so individualized diagnosis and treatment options are common.

Required Author Forms Disclosure forms provided by the author are available with the online version of this article.

\section{References}

1. Caviness JN, Brown P. Myoclonus: current concepts and recent advances. Lancet Neurol 2004;3:598-607.

2. Marsden CD, Hallett M, Fahn S. The nosology and pathophysiology of myoclonus. In: Marsden CD, Fahn S (eds) Movement disorders. Butterworths, London, 1983, pp. 196-248.

3. Kinugawa K, Vidailhet M, Clot F, Apartis E, Grabli D, Roze E. Myoclonus-dystonia syndrome: an update. Mov Disord 2009;24: 479-489. 
4. Caviness JN, Alving LI, Maraganore DM, et al. The incidence and prevalence of myoclonus in Olmsted County, Minnesota. Mayo Clin Proc 1999;74:565-569.

5. Caviness JN. Treatment of myoclonus. In: UpToDate. Available at: www.UpToDateInc.com. Accessed April 8, 2013.

6. Dijk JM, Tijssen MAJ. Management of patients with myoclonus: available therapies and the need for an evidence-based approach. Lancet Neurol 2010;9:1028-1036.

7. Shibasaki H. Electrophysiologic studies of myoclonus. Muscle Nerve 2000;23:321-335

8. Zifkin B, Andermann E, Andermann F. Mechanisms, genetics, and pathogenesis of juvenile myoclonic epilepsy. Curr Opin Neurol 2005; 18:147-153.

9. Snead OC. Basic mechanisms of generalized absence seizures. Ann Neurol 1995;37:146-57.

10. Savic I, Pauli S, Jan-Olof T, et al. In vivo demonstration of altered benzodiazepine receptor density in patients with generalized epilepsy. J Neurol Neurosurg Psychiatr 1994;57:797-804.

11. Deppe M, Kellinghaus C, Duning T, et al. Nerve fiber impairment of anterior thalamocortical circuitry in juvenile myoclonic epilepsy. Neurology 2008;71:1981-1985.

12. Caviness JN. Clinical neurophysiology of myoclonus. In: Hallett M (ed.) Movement disorders. Handbook of clinical neurophysiology. Elsevier, Amsterdam, 2003, pp. 521-548.

13. Caviness JN. Epileptic myoclonus. In: Sirven JI, Stern JM (eds) Atlas of video-EEG monitoring. McGraw-Hill Medical, New York, 2011, pp 309-328.

14. Roze E, Apartis E, Clot F, et al. Myoclonus-dystonia: clinical and electrophysiologic pattern related to SGCE mutations. Neurology 2008;70(13):1010-1016.

15. Gwinn KA, Caviness JN. Electrophysiological observations in idiopathic opsoclonus-myoclonus syndrome. Mov Disord 1997;12(3): 438-442.

16. Chadwick D, French AT. Uraemic myoclonus: an example of reticular reflex myoclonus? J Neurol Neurosurg Psychiatry 1979;42:5255.

17. Caviness JN. Segmental myoclonus. In: Albanese A, Jankovic J (eds) Hyperkinetic movement disorders: differential diagnosis and treatment. Wiley-Blackwell, Chichester, 2012, pp. 221-235.

18. Calancie B. Spinal myoclonus after spinal cord injury. J Spinal Cord Med 2006;29:413-424.

19. Keswani SC, Kossoff EH, Krauss GL, et al. Amelioration of spinal myoclonus with levetiracetam. J Neurol Neurosurg Psychiatry 2002;73:457-458

20. Evidente VGH, Caviness JN. Focal cortical transient preceding myoclonus during lithium and tricyclic antidepressant therapy. Neurology 1999;52:211-213.

21. Jang W. Kim JS. Ahn JY. Kim HT. Reversible propriospinal myoclonus due to thoracic disc herniation: long-term follow-up. J Neurol Sci 2012; 313:32-34.

22. Witte OW, Niedermeyer E, Arendt G, Freund HJ. Post-hypoxic action (intention) myoclonus: a clinic-electroencephalographic study. J Neurol 1988;235:214-218.

23. Brown P, Steiger MJ, Thompson PD, et al. Effectiveness of piracetam in cortical myoclonus. Mov Disord 1993;8:63-68.

24. Ikeda A, Shibasaki H, Tashiro K, et al. Clinical trial of piracetam in patients with myoclonus: nationwide multiinstitution study in Japan. Mov Disord 1996;11:691-700.

25. Koskiniemi M, Vleymen B, Hakamies L, et al. Piracetam relieves symptoms in progressive myoclonus epilepsy: a multicentre, randomized, double blind, crossover study comparing the efficacy and safety of three dosages of oral piracetam with placebo. J Neurol Neurosurg Psychiatr 1998;64:344-348.

26. Fedi M, Reutens D, Dubeau F, et al. Long-term efficacy and safety of piracetam in the treatment of progressive myoclonus epilepsy. Arch Neurol 2001;58:781-786.
27. Frucht SJ, Louis ED, Chuang C, et al. A pilot tolerability and efficacy study of levetiracetam in patients with chronic myoclonus. Neurology 2001;57:1112-1114.

28. Genton P, Gelisse P. Antimyoclonic effect of levetiracetam. Epileptic Disord 2000;2:209-212.

29. Tai KK. Truong DD. Brivaracetam is superior to levetiracetam in a rat model of post-hypoxic myoclonus. J Neural Transm 2007;114:15471551.

30. Kalviaiinen R, Genton P, Andermann E, et al. Brivaracetam in patients with Unverricht-Lundborg disease, results from two randomized, placebo-controlled, double-blind studies. Epilepsia 2009;50:47.

31. VanDongen AMJ, VanErp MG, Voskuyl RA. Valproate reduces excitability by blockage of sodium and potassium conductance. Epilepsia 1986;27:177-182.

32. Obeso JA, Artieda J, Rothwell JC, et al. The treatment of severe action myoclonus. Brain 1988;112:765-777.

33. Silver M, Factor SA. Valproic acid-induced parkinsonism: Levodopa responsiveness with dyskinesia. Parkinsonism Relat Disord 2013;19: $758-760$.

34. Meldrum BS. Drugs acting on amino acid neurotransmitters. Adv Neurol 1986;43:687-706.

35. Jenner P, Pratt JA, Marsden CD. Mechanism of Action of clonazepam in myoclonus in relation to effects on GABA and 5-HT. Adv Neurol 1986;43:629-643.

36. Wagner HR, Reches A, Yablonskaya E, Fahn S. Clonazepaminduced up-regulation of serotonin1 and serotonin2 binding sites in rat frontal cortex. Adv Neurol 1986;43:645-660.

37. Kyllerman M, Ben-Menachem E. Zonisamide for progressive myoclonus epilepsy: long-term observations in seven patients. Epilepsy Res 1998;29:109-114.

38. Frucht SJ, Houghton WC, Bordelon Y, et al. A single-blind, openlabel trial of sodium oxybate for myoclonus and essential tremor Neurology 2005;65:1967-1970.

39. Hosford DA, Caddick SJ, Fu-hsiung L. Generalized epilepsies: emerging insights into cellular and genetic mechanisms. Curr Opin Neurol 1997;10:115-120.

40. Blumenfeld H. From molecules to networks: cortical/subcortical interactions in the pathophysiology of idiopathic generalized epilepsy. Epilepsia 2003;44(Suppl. 2):7-15.

41. Duron RM, Medina MT, Martinez-Juarez IE, et al. Seizures of idiopathic generalized epilepsies. Epilepsia 2005;46(Suppl. 9):34 47.

42. Wallace SJ. Myoclonus and epilepsy in childhood: A review of treatment with valproate, ethosuximide, lamotrigine and zonisamide. Epilepsy Res 1998;29:147-154.

43. Christe W, Jacob R, Janz D. Juvenile myoclonic epilepsy: response to valproate monotherapy in 27 previously untreated patients. Epilepsia 1995;36(Suppl. 3):S65.

44. Calleja S, Salas-Puig J, Ribacoba R, et al. Evolution of juvenile myoclonic epilepsy treated from the outset with sodium valproate. Seizure 2001;10:424-427.

45. Covanis A, Gupta AK, Jeavons PM. Sodium valproate: monotherapy and polytherapy. Epilepsia 1982;23:693-720.

46. Buchanan N. The use of lamotrigine in juvenile myoclonic epilepsy. Seizure 1996;5:149-151.

47. Crespel A, Genton P, Berramdane M, et al. Lamotrigine associated with exacerbation or de novo myoclonus in idiopathic generalized epilepsies. Neurology 2005;65:762-764.

48. Parmeggiani L, Seri S, Bonanni P, et al. Electrophysiological characterization of spontaneous and carbamazepine-induced epileptic negative myoclonus in benign childhood epilepsy with centro-temporal spikes. Clin Neurophysiol 2004;115:50-58.

49. Wheless, JW, Sankar, R. Treatment strategies for myoclonic seizures and epilepsy syndromes with myoclonic seizures. Epilepsia 2003;44(Suppl. 11):27-37. 
50. Sheth RD, Gidal BE. Intravenous valproic acid for myoclonic status epilepticus. Neurology 2000;54:1201.

51. Pellock, JM. Myoclonic epilepsies: syndromes and their treatment. Suppl Clin Neurophysiol 2004;57:425-432.

52. Medina, MT, Martinez-Juarez IE, Duron RM, et al. Treatment of myoclonic epilepsies of childhood, adolescence, and adulthood. Adv Neurol 2005;95:307-323.

53. Sankar R, Wheless JW, Dravet C, et al. Treatment of myoclonic epilepsies in infancy and early childhood. Adv Neurol 2005;95: 289-298.

54. Caviness JN, Forsyth PJ, McPhee T, Layton DD. The movement disorder syndrome of adult opsoclonus. Mov Disord 1995;10:22-27.

55. Obeso JA. Therapy of myoclonus. Clin Neurosci 1995-1996;3:253257.

56. Rughani AI, Lozano AM. Surgical treatment of myoclonus dystonia syndrome. Mov Disord 2013;28:282.

57. Aysun U, Sumer M, Atasoy T, Nuray A. Treatment of idiopathic opsoclonus-myoclonus with intravenous immunoglobulin. Neurol India 2004;52(4):520-521.

58. Anderson NE, Budde-Steffen C, Rosenblum MK, et al. Opsoclonus, myoclonus, ataxia, and encephalopathy in adults with cancer: a distinct paraneoplastic syndrome. Medicine 1988;67:100-109.

59. Digre KB. Opsoclonus in adults. Arch Neurol 1986;43:1165-1175.

60. Harada Y, Ishmimitsu H, Nishimoto K, Miyata I, Nobuhiko M. Opsoclonus-polymyoclonia syndrome suppressed with reserpine. Brain Nerve 1986;38:359-362.

61. Chadwick D, Hallett M, Harris R, Jenner P, Reynolds EH, Marsden CD. Clinical, biochemical and physiological factors distinguishing myoclonus responsive to 5-hydroxytryptophan, tryptophan plus a monoamine oxidase inhibitor, and clonazepam. Brain 1977;100: 455-487.

62. Roze E, Bounolleau P, Ducreux D, et al. Propriospinal myoclonus revisited: clinical, neurophysiologic, and neuroradiologic findings. Neurology 2009;72:1301.

63. Fouillet N, Wiart L, Arne P, Alaoui P, Petit H, Barat M. Propriospinal myoclonus in tetraplegic patients: clinical, electrophysiological and therapeutic aspects. Paraplegia 1995;33:678-681.

64. Van der Salm SMA, Koelman JHTM, Henneke S, van Rootsdelaar AF, Tijssen MAJ. Axial jerks: a clinical spectrum ranging from propriospinal to psychogenic myoclonus. J Neurol 2010;257:1349-1355.
65. Jankovic J, Pardo R. Segmental myoclonus. Clinical and pharmacologic study. Arch Neurol 1986;43:1025-1031.

66. Wakata N, Sugimoto H, Iguchi H, et al. A case of voluntary palatal myoclonus with ear click: relationship between palatal myoclonus and click. Eur Neurol 2002;48:52-53.

67. Fabiani G, Teive HA, Sa D, et al. Palatal myoclonus: report of two cases. Arq Neuropsiquiatr 2000;58:901-904.

68. Scott BL, Evans RW, Jankovic J. Treatment of palatal myoclonus with sumatriptan. Mov Disord 1996;11:748-751.

69. Nasr A, Brown N. Palatal myoclonus responding to lamotrigine. Seizure 2002;11:136-137.

70. Ensink RJH, Vingerhoets HM, Schmidt CW, et al. Treatment for severe palatoclonus by occlusion of the eustachian tube. Otol Neurotol 2003;24:714-716.

71. Krause E, Leunig A, Klopstock T, et al. Treatment of essential palatal myoclonus in a 10 -year-old girl with botulinum neurotoxin. Otol Neurotol 2006;27:672-675.

72. Srirompotong S, Tiamkao S, Jitpimolmard S. (2002) Botulinum toxin injection for objective tinnitus from palatal myoclonus: a case report. J Med Assoc Thai 2002;85:392-395.

73. Penney SE, Bruce IA, Saeed SR. Botulinum toxin is effective and safe for palatal tremor: a report of five cases and a review of the literature. J Neurol 2006;253:857-860.

74. Keswani SC, Kossoff EH, Krauss GL, et al. Amelioration of spinal myoclonus with levetiracetam. J Neurol Neurosurg Psychiatry 2002;73:457-458.

75. Estraneo A, Saltalamacchia AM, Loreto V. Spinal myoclonus following herpes zoster radiculitis. Neurology 2007;68:E4.

76. Lagueny A, Tison G, Burbaud P, et al. Stimulus-sensitive spinal segmental myoclonus improved with injections of botulinum toxin type A. Mov Disord 1999;14:182-185.

77. Valls-Sole J. Electrodiagnostic studies of the facial nerve in peripheral facial palsy and hemifacial spasm. Muscle Nerve 2007;36:14-20.

78. Carnero-Pardo C, Sanchez-Alvarez JC, Gomez-Camello A, et al. Myoclonus associated with thoracodorsal neuropathy. Mov Disord 1998;13(6):971-972.

79. Hallett M. Physiology of psychogenic movement disorders. J Clin Neurosci 2010;17:959-965.

80. Czarnecki K, Hallett M. Functional (psychogenic) movement disorders. Curr Opin Neurol 2012;25:507-512. 